\title{
Optimal Conditions for Defect Analysis Using Electron Channeling Contrast Imaging
}

\author{
Jin-Su Oh, Cheol-Woong Yang* \\ School of Advanced Materials Science and Engineering, Sungkyunkwan University, Suwon 16419, Korea
}

*Correspondence to:

Yang CW,

Tel: +82-31-290-7362

Fax: +82-31-290-7371

E-mail: cwyang@skku.edu

Received September 26, 2016 Revised September 26, 2016

Accepted September 26, 2016
Electron channeling contrast imaging (ECCI) is a powerful analyzing tool for identifying lattice defects like dislocations and twin boundaries. By using diffraction-based scanning electron microscopy technique, it enables microstructure analysis, which is comparable to that obtained by transmission electron microscopy that is mostly used in defect analysis. In this report, the optimal conditions for investigating crystal defects are suggested. We could obtain the best ECCI images when both acceleration voltage and probe current are high (30 kV and $20 \mathrm{nA})$. Also, shortening the working distance $(6 \mathrm{~mm})$ enhances the quality of defect imaging.

Key Words: Scanning electron microscopy, Electron channeling contrast imaging, Defect analysis

\section{INTRODUCTION}

The crystal defect analyses have been commonly conducted using transmission electron microscopy (TEM). There is no doubt that TEM is one of the best analyzing tool for examining crystal defects but limitations are bound to follow due to the shape of specimen; the thin foil (Williams \& Carter, 2009). Recently, scanning electron microscopy (SEM) based technique for defect analysis, which is called electron channeling contrast imaging (ECCI), has got popularity. As a non-destructive and diffraction-based method, ECCI could take some advantages over analysis using TEM (Carnevale et al., 2015). In the first, the specimen preparation is relatively simple and only requires one side of a flat surface. It could exclude the concerns about whether defects to analyze are caused by the process of the specimen preparation or not. In addition, inspecting large area of flat surface could ensure the representativeness of the specimen.

It is important to meet the exact Bragg-diffraction condition in order to distinguish dislocation lines from the background matrix (Simkin \& Crimp, 1999). In the SEM, there are two approaches to achieve this condition. One is selected area channeling pattern (SACP), and the other is electron backscatter diffraction (EBSD). SACP could provide the rocking beam to induce electron channeling patterns (ECPs) in a small grain. With tilting the specimen, ECPs of the specific $h k l$-plane could move to meet the optic axis, i.e., the exact Bragg-diffraction condition. Despite its great angular accuracy, SACP has the poor resolution (Lloyd, 1987). Therefore it is almost impossible to conduct SACP at a few or sub-micrometer sized grain. The latter, EBSD, could overcome this situation. But it has its own limitation; poor angular accuracy. So we have to use both techniques to determine the Bragg-condition complementarily.

\section{EXPERIMENTAL}

In the present study, deformation of pure nickel induced by shot peening was examined by SEM (JSM-7000F; JEOL Co., Ltd., Japan) equipped with a field emission gun. The surface of the shot peened nickel was prepared by mechanical polishing with $0.02 \mu \mathrm{m}$ colloidal silica.

(a) This is an open-access article distributed under the terms of the Creative Commons Attribution Non-Commercial License (http://creativecommons.org/licenses/by-nc/4.0) which permits unrestricted noncommercial use, distribution, and reproduction in any medium, provided the original work is properly cited.

Copyrights @ 2016 by Korean Society of Microscopy 

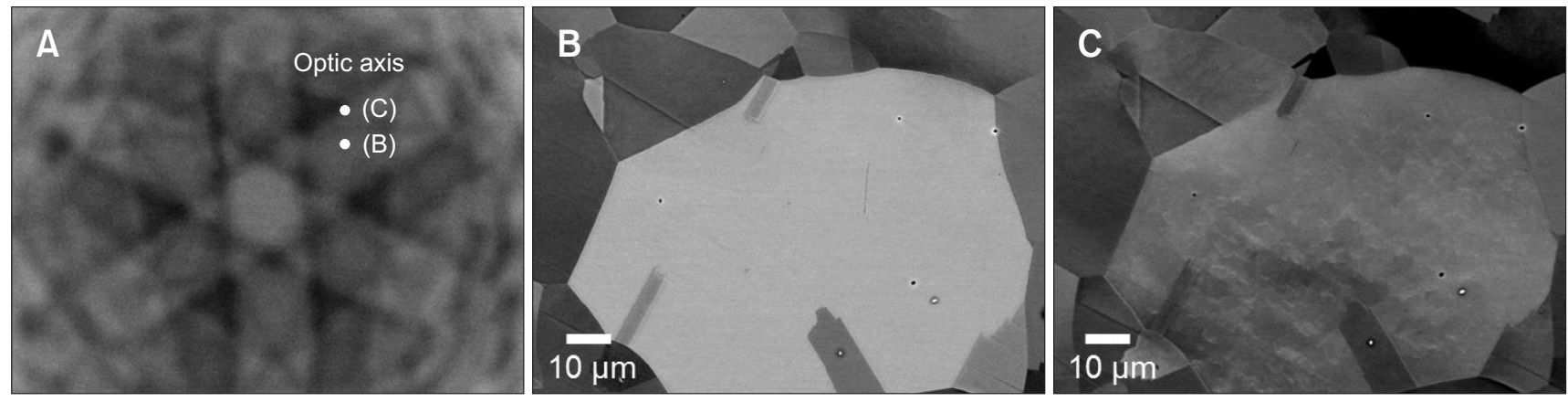

Fig. 1. (A) Electron channeling pattern in a grain. The optic axis moves when the specimen is tilting. When the optic axis is at (B), which means that the deviation parameter $\mathbf{s}$ is smaller than zero, defects in the grain are almost undetectable. When the optic axis is at (C); under the exact Bragg-diffraction condition, however, the defects become distinguishable from the background matrix.
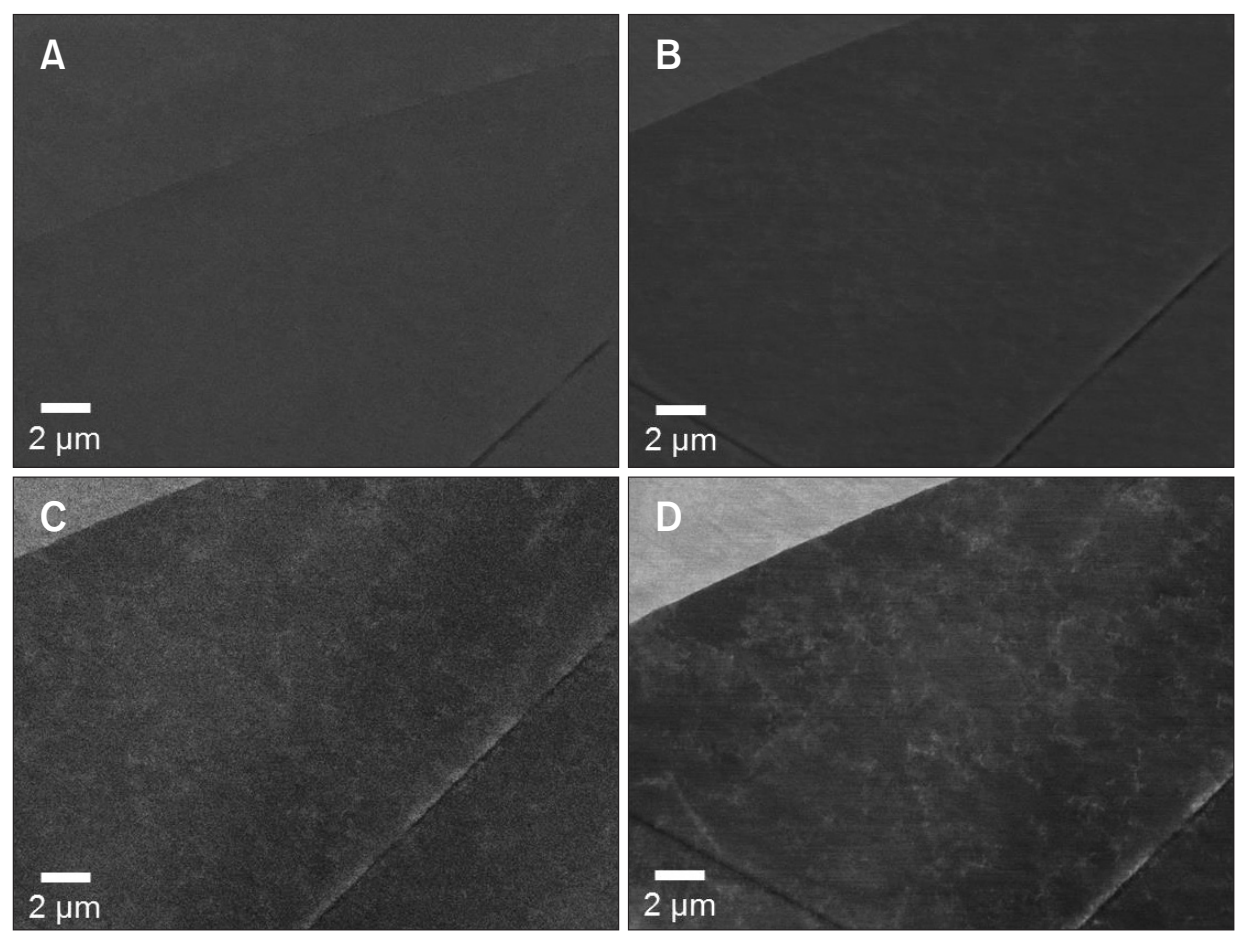

Fig. 2. Electron channeling contrast images obtained under the various combinations of acceleration voltage and probe current. (A) $20 \mathrm{kV}, 3 \mathrm{nA}$. (B) $20 \mathrm{kV}$, 20 nA. (C) 30 kV, 3 nA. (D) 30 kV, 20 nA. It is clearly shown that high acceleration voltage and high probe current could provide even clearer contrast.
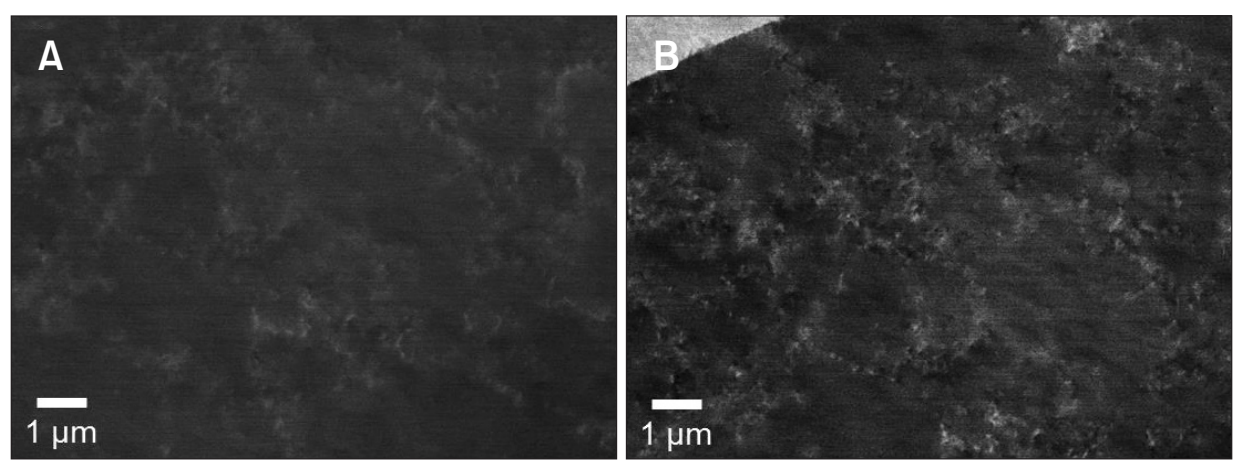

Fig. 3. The change of contrast with changing the working distance. Electron channeling contrast imaging (ECCI) images obtained under the condition of working distance of $8 \mathrm{~mm}(\mathrm{~A})$ and $6 \mathrm{~mm}$ (B). When we just shorten the working distance and other variables are held, the quality of ECCI image can be enhanced.

\section{RESULTS AND DISCUSSION}

As shown in Fig. 1A, SACP was carried out within a single grain. A clear ECP was obtained by careful eucentric correction. By tilting the specimen, the exact Braggdiffraction condition can be met. At this time, much higher contrast induced by crystal defects was observed even at low magnification (Fig. 1B and C). 
Next, we compare the images under various acceleration voltages and probe currents. ECCI images were obtained under specific conditions; an acceleration voltage of 20 to $30 \mathrm{kV}$ and a probe current of 3 to $20 \mathrm{nA}$. Of course, a high acceleration voltage gives rise to good image resolution and a high probe current also increase the yield of detected backscattered electron so it leads to better image quality. Among those conditions, a combination of the highest acceleration voltage $(30 \mathrm{kV})$ and the highest probe current $(20$ $\mathrm{nA}$ ) exhibited the clearest ECCI image with a high contrast (Fig. 2).

In addition, the effect of working distance on the ECCI image was investigated by varying it from 6 to $8 \mathrm{~mm}$. It is recommended to use a working distance as short as possible. Backscattered electrons are always insufficient. By shortening the working distance, more backscattered electrons could be collected and therefore it leads to the higher contrast, as clearly seen in Fig. 3. However, extreme care should be taken when you shorten the working distance, because the specimen could hit the backscattered electron detector. In this work, the optimum working distance was determined to be $6 \mathrm{~mm}$.

\section{SUMMARY}

In summary, with this state-of-the art SEM technique, the crystal defects such as dislocations and twin boundaries are easily and clearly observed. A high acceleration voltage and a high probe current enhance the quality of the ECCI image and so does a short working distance. Under the optimal condition described here, the defect analysis can be carried out more efficiently. With the combination of EBSD, its applications can be more extensive.

\section{CONFLICT OF INTEREST}

No potential conflict of interest relevant to this article was reported.

\section{ACKNOWLEDGMENTS}

This work was partially supported by NRF grants by the Korean government (MSIP and MOE) (NRF-2011-0030058 and NRF-2015R1D1A1A01059653), and in part by the National Research Council of Science \& Technology (NST) grant by the Korea government (MSIP) (No. CRC-15-06KIGAM).

\section{REFERENCES}

Carnevale S D, Deitz J I, Carlin J A, Picard Y N, McComb D W, DeGraef M, Ringel S A, and Grassman T J (2015) Applications of electron channeling contrast imaging for the rapid characterization of extended defects in III-V/Si heterostructures. IEEE J. Photovolt. 5, 676-682.

Lloyd G E (1987) Atomic number and crystallographic contrast images with the SEM: a review of backscattered electron techniques.
Mineral. Mag. 51, 3-19.

Simkin B A and Crimp M A (1999) An experimentally convenient configuration for electron channeling contrast imaging. Ultramicroscopy 77, 65-75.

Williams D B and Carter C B (2009) Transmission Electron Microscopy: A Text Book for Materials Science (Springer, New York). 